\title{
Evaluation of tunneled tunica vaginalis flap (TVF) used as adjunct in the repair of urethrocutaneous fistula post tubularized incised plate urethroplasty (TIP) technique
}

\author{
M Khalifa, MD; I Tantawy, MD; Amera H Waly, MD; Salah M Abdelaal MD \\ Department of Pediatric Surgery, Zagazig University Hospital, Egypt.
}

\begin{abstract}
Background and purpose: Urethrocutaneous fistula (UCF) remains the most frequent encountered complication of hypospadius surgery even in experienced surgical hands. The key measure to ensure a successful repair is separation of the suture lines in the urethra and skin, using well vascularized elastic tissue. This study aims to use tunneled tunica vaginalis flap(IVF) as adjunct in UCF repair either initial or recurrent following TIP technique and to evaluate outcome of cases regarding recurrence of fistula and other postoperative complications.

Material and methods: In period between Jun. 2007 to Jun.2009, 14 cases of urethrocutaneous fistula post TIP technique with a mean age 5.6 year (3-11) were operated on using TVF. For each patient, we reported original preoperative location of urethral meatus, the number and location of fistulas, number of surgery, size of fistula and duration of surgery. Outcome of cases regarding to recurrence of fistula and other complications was reported. Follow up of cases ranged.from 6 months to 24 months (mean 12 months).

Result: Of total 14 patients, $5(35.7 \%)$ patients had previous attempt for closure of UCF ranged.from 1-2 times. 4 (28.6\%) patients had multiple UCF ranged.from 2-3 fistulae at different location. Site of UCF was maximally located proximal penile followed by midpenile. Size of UCF ranged from 2 to $10 \mathrm{~mm}$. Operative time ranged from 45 to 90 minutes with a mean time (60) minutes.Duringfollow up period no delayed postoperative complications had been observed.

Conclusion: The tunneled tunica vaginalis flap is an excellent method for closure of both initial and recurrent urethrocutaneous fistula post TIP technique. The flap is easy to be harvested and mobilized without testicular complication and provide sufficient length to cover repaired UCF regardless of number, location and size of fistula.
\end{abstract}

Key words: Hypospadius surgery, tubularized incised plate urethroplasty, urethrocutaneous fistula, tunica vagina/is flap.

\section{Introduction:}

Hypospadias surgery has evolved with more than 150 procedures described for surgical correction of a single anomaly) The most significant recent advance in surgical technique occurred in 1994 with Snodgrass' description of the tubularized incised plate (TIP) urethroplasty technique.2 Despite these advances in urethroplasty technique, a review of TIP urethroplasty reveals a combined UCF rate of $2.4 \%$ across several major centers study. The majority of fistulas occurred at the junction of the neourethra with the urethra. 3

Surgery for urethrocutaneous fistulas has remained a challenge for the treating surgeons and several surgical procedures have been described each claiming good results. Factors that may affect results of UCF repair may be the conditions of local tissue, duration of time after hypospadias repair, the number, location and size of the fistula, use of magnification, patient's age, previous fistula repairs and also the type of suture material used, skill of the operating surgeon and proper inversion of the edges etc. The key measure to ensure a successful repair is separation of the suture lines in the urethra and skin, using well vascularized elastic tissue. 4

In the conventional simple repair of urethrocutaneous fistulae, three layers of tissue are mobilized (margins of urethrocutaneous fistula, Dartos/surrounding tissue, skin) and 
closed using fine absorbable sutures, However, this can be difficult to achieve a wellvascularized tissues amenable to interposition $s$

One possibility for interposition graft for UCF repair is the use of tunneled TVF. Unlike dissection of Dartos layer which can damage blood supply of overlying skin with impaired wound healing, tunica vaginalisbrings vascular supply from outside source hence helping in healing of suture line of UCF repair.6

Material and method:

In peroid between Jun. 2007 to Jun. 2009, 14 cases of urethrocutaneous fistula post TIP technique were admitted at our pedaitric surgical unit for repair of UCF (9 initial and 5 recurrent) with at least 6 -month period between the previous surgery and UCF repair for achievement of healing. Age of patients ranged from 3 to 11years with a mean age of (5.6year).

For each patient, we reported original preoperative location of urethral meatus, the number and location of fistulas, number of surgery, size of fistula, duration of surgery, length of follow up and postoperative complications including wound infection, scrotal hematoma, testicular tethering, penile torsion or recurrence of fistulae.

In all 14 cases tunneled tunica vaginalis flap was used as adjunct in UCF repair. Outcome of cases regarding recurrence of fistula and other complications was reported.

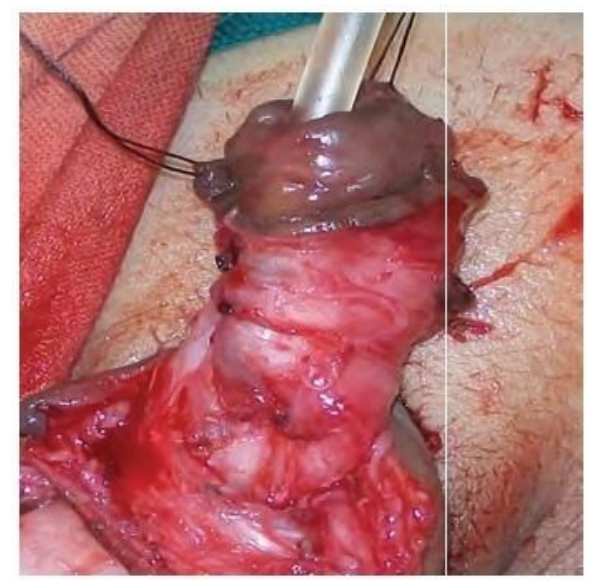

Figure (1): Closure of multiple urethroctaneous fistula, one penoscrotal, one distal penile and one subcoronal.
Follow up of cases ranged from 6 months to 24 months with a mean of (12 months). Operative tec:hnique:

All patients were operated on undergeneral anesthesia. Urethral calibration was done to exclude distal obstruction. Dilute povidone solution was injected through the meatus to visualize exact location and number of fistula. Circumferential skinincision was done around UCF and skin was mobilized and undermined to expose fistula. In case of multiple UCF at different location proximal and distal, penile skin degloving with careful dissection was performed. UCF1ract was excised and urethra was closed in two layer using 6/0 vicryl sutures over catheter Figure(!). All repairs were checked for watertight anastomosis by injecting saline into the urethra by syring through meatus. Small incision 1-2 em was done high in the anterior wall of hemiscrotum and scrotal attachment was dissected from tunica vaginalis. Testis was delivered from scrotal incision. The TVF was harvested on vascular pedicle and separated from testicle and cord Figure(2). The length of flap is adjusted according to the distance from harvested site to UCF site. The TVF is then tunneled underneath the penile skin Flgures $(3,4)$. The TVF is then fixed at each UCF site using 6/0 vicryl Figure(5). Scrotal incision was closed without drain after reduction of testicle inits place and penile skin incision was closed to complete procedure Figure(6). Urinary catheter was faxed for 710 days.

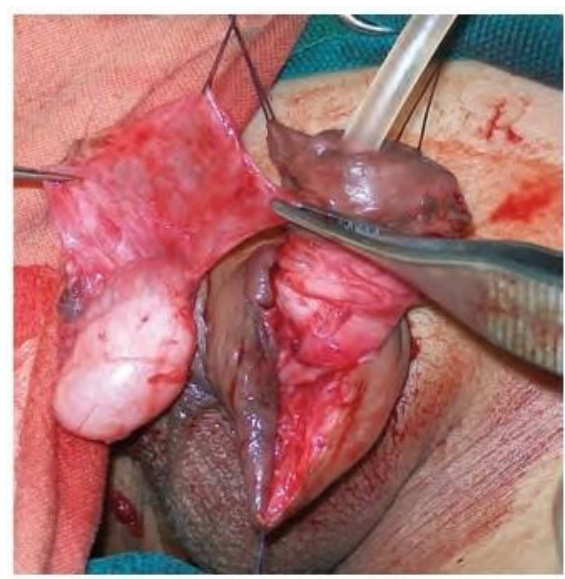

Figure (2): The TVFwas harvested on vascular pedicle and separated from testis and cord. 

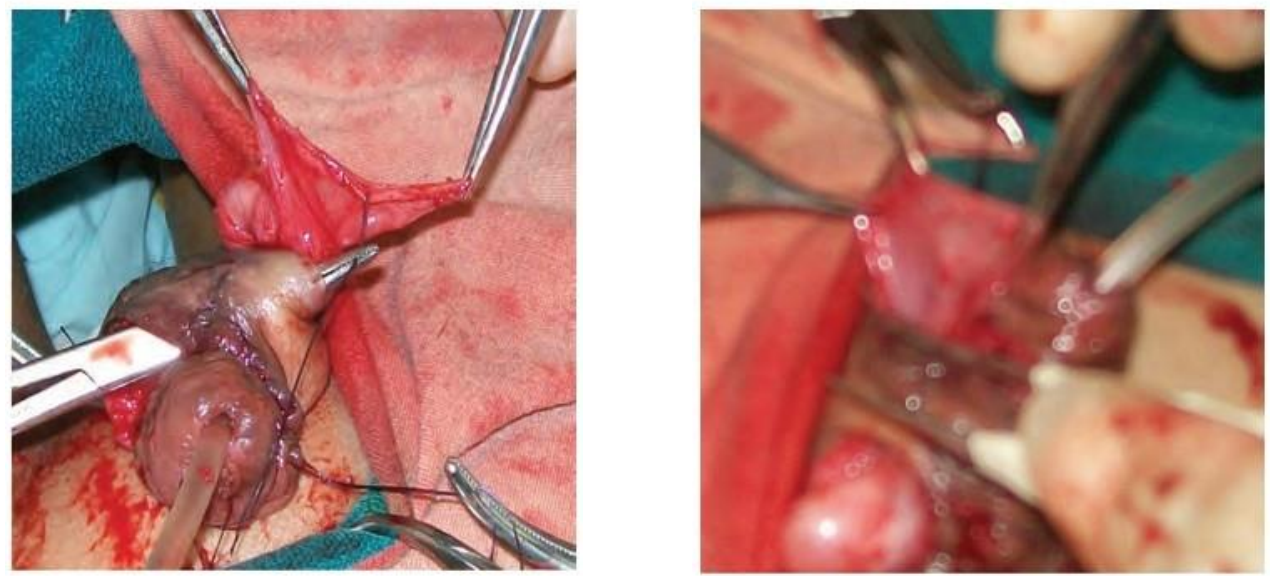

Figures (3) and (4): The TVF is tunneled underneath the penile skin

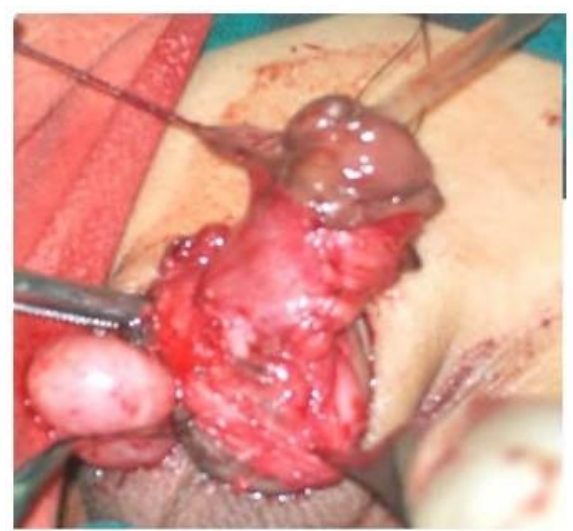

Figure (5): Fixation of tunica vagina/is flap.

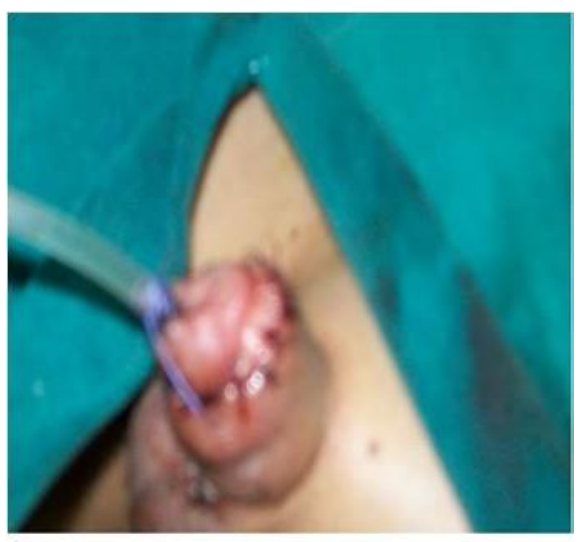

Figure (6): Closure of penile and scrotal incision and final appearance of technique.

Table (1): Types of hypospadius.

\begin{tabular}{|l|c|}
\hline Site & Number \\
\hline Penoscrotal & $3(21.4 \%)$ \\
\hline Proximal penile & $5(35.7 \%)$ \\
\hline Midpenile & $4(28.6 \%)$ \\
\hline Distal penile & $2(14.3 \%)$ \\
\hline Subcoronal & $0(0 \%)$ \\
\hline
\end{tabular}

Table(2): Site ofUCF.

\begin{tabular}{|l|l|}
\hline Site of fistula & Number \\
\hline Penoscrotal & $3(15 \%)$ \\
\hline Proximal penile & $6(30 \%)$ \\
\hline Midpenile & $5(25 \%)$ \\
\hline Distal penile & $4(20 \%)$ \\
\hline Subcoronal & $2(10 \%)$ \\
\hline
\end{tabular}




\section{Discussion:}

Among the different surgical procedures advocated in hypospadias repair, urethrocutaneous fistula remains the most frequent encountered complication and a serious problem, even in experienced surgical hands.7 Many innovations have led to advances in the surgical repair ofhypospadius.4 One of the most recent modifications is the tubularized incised plate (TIP) urethroplasty described by Snodgrass in 1994,2 which has become one of the most commonly used techniques in hypospadias repair. The complication rate associated with TIP urethroplasty is $5-10 \%$, with the majority of complications being urethrocutaneous fistulae. 8 Other techniques have been associated with fistula rates up to $20 \% .9$

Urethrocutaneous fistulae (UCF) usually result following loss of viability of tissues involved in hypospadias repair.10 Necrosis of overlying skin subcutaneous tissue leaves the neo-urethra exposed, thus resulting in either its total or partial disruption or fistula formation. Also, excessive backpressure due to distal obstruction can disrupt adequately reinforced repairs of good integrity.11

The different techniques recommended in the available literature with the aim to reduce the incidence ofUCF includes: avoidance of a opposing urethral and skin sutures, use of fine scalpel for skin incision, minimal tissue trauma by use of fine forceps or hooks and an inverting watertight mucosal suturing. 12 Better suture materials, use of magnification, dorsal subcutaneous flap and silicone catheters have all contributed to a decrease in fistula rate. 13

The role of a waterproofmg layer between the urethra and skin is well established in the prevention and repair of fistula complication in hypospadias surgery. The common waterproofing techniques utilize subcutaneous tissue, Dartos fascia, Dartos muscle from scrotum and tunica vaginalis from the scrotum. 6

Tunneled TVF, was first described in 1970 by Hosli14 and subsequently popularized by Snow et al.15 Shankar et al.16 operated 10 cases of recurrent UCF and limited the use of tunica vaginalis as waterproofing layer to the third or subsequent repair. Routh et aJ17 had used tunneled tunica vaginalis flap for 16 patients who had failed previous UCF repair post TIP technique. In our study, tunneled tunica vaginalis flap was used for both initial UCF (72.4\%) and recurrent UCF (28.6\%). It is clear that with subsequent attempts at fistula repair, the chances of recurrence increases with decrease in success rates owing to the further scarring of the already deficient compromised surrounding skin.

A series of 160 patients which had undergone urethrocutaneous fistula repair after hypospadias surgery were studied and the prognostic significance of the site, size, number of fistulas and number of the previous operations, they concluded that there was no impact of these factors on success rate. 18 In this study tunneled TVF was done for all our patients regardless site, number and size of fistula.

A common error observed is timing of fistula repair. Consensus and logic in this regard dictates a wait and see policy for at least six months of last repair to enable the scars to mature and also the oedema and indurations to subside.13 In this study we operated all patients after at least six months from last operation.

The TVF is a good vascularized flap, as it has a different blood supply and does not depend on the vascularity of penile skin, unlike the Dartos fascia.19 It has a dependable blood supply from the cremasteric vessels and its pedicle length can safely be increased up to the external inguinal ring.6 Dissecting the TVF is technically easy and may be done even by beginners in hypospadius surgery.19 In the present study, we did not face any difficulty in harvesting the TVF from the testis and the cord and gained a sufficient length to cover the UCF regardless the location or number of fistula. Care must be taken while increasing pedicle length as the tissue becomes more flimsy proximally and at the same time inadequate pedicle length can cause tethering of the testis at a higher level compared to the normal side in an erect position 6 Inthis study, no case of testicular tethering occurred during the follow up period.

Muruganandham et al20 operated on 51 patients of UCFS by 3 different surgical technique, 21 of them had been operated using 
TVF and did not report any postoperative complication regarding to recurrence of fistula, penile torsion and no postoperative complications were encountered in testis or scrotum. Similar results had been obtained by Routh et al17 in their series of 16 boys with previous failed attempt UCF repair. In the present study, no cases of recurrence of fistula were reported during follow up period. Immediately postoperative, there was one case who developed small scrotal haematoma which resolved spontaneously. One case had wound healing problems because of skin sloughing and wound dehiscence at suture line but had completed healing without recurrence of fistula because of vascular tunica vaginalis interposition flap acting as barrier.

\section{Conclusion:}

The tunneled tunica vaginalis flap is an excellent method for closure of both initial and recurrent urethrocutaneous fistula post TIP technique.The flap is easy to be harvested and mobilized without testicular complication and provides sufficient length to cover repaired UCF regardless of number, location and size of fistula.

\section{References:}

1- HodgsonNB:History ofhypospadias repair, Reconstructive and plastic surgery of the external genitalia adult and pediatric. WB Saunders Company 1999; p.16.

2- Snodgrass W: Tubularized, incised plate urethroplasty for distal hypospadias. Journal ofUrology 1994; 151(2):464-465.

3- Snodgrass WT: Tubularized incised plate hypospadias repair: Indications, technique and complications. Urology 1999; 54(1): 6-11.

4- Soni A, Sheoran S: Repair of large urethrocutaneous fistula with Dartos-based flip flap: A study of 23 cases. Indian J Plas Surg 2007; 40(1): 34-38.

5- Sarkar R, Konar H, Sahabasu K, Saha K, Samanta N: Urethroplasty by midline longitudinal incision of the dorsal wall of urethra at the site of the fistula: A useful adjunct to the conventional method of repair ofurethrocutaneous fistula developing after hypospadias surgery. Journal of Indian
Association of Pediatric Surgeons 2006; 11(1): 24-26.

6- Handoo YR: Role of tunica vaginalis interposition layer in hypospadias surgery. Indian J Past Surg 2006; 39(2): 152-156.

7- Kargi E, Yessili C, Akduman B, Babuccu O, Hosnuter M, Mungan A: Fascia lata grafts for closure of secondary urethral fistulas. Urology 2002; 62: 928-931.

8- Duckett JW, Baskin L: Hypospadias. In: Adult and pediatric urology. Gillenwater JY, Grayhack JT, Howards SS, et al (Editors); St. Louis: Mosby-Year Book (Publisher); 3rd edn. Vol. 3. 1998. p. 2549 [Chapter 55].

9- Kass EJ, Bolong D: Single-stage hypospadias repair without fistula. J Urol 1990; 144: 520.

10-Singh RB, Pavithran NM: Partially deepithelialized preputial flap (triangular soft tissue flap): An aid to prevent coronal urethrocutaneous fistulae. Pediatr Surg Int 2003; 19: 551-553.

11-Singh RB, Dalal S, Pavithran NM, Sharma BD:Soft tissue reinforcement interposition flaps in hypospadias repair. Indian J Plast Surg 2007; 40(2).

12-Iatifogui O, Yaveizer R, Unal S: Surgical treatment of urethral fistulas following hypospadias repair. Ann Plast Surg 2000; 44:381.

13-Retic AB, Keating M, Mandell J: Complications ofhypospadias repair. Urol Clin North Am 1988; 15: 223-226.

14-Hosli PO:Eine technik zum verschluss von harnrohrenfistein. Urologe 1970; 9:129.

15-Snow BW: Use of tunica vaginalis to prevent fistulas in hypospadias surgery. The journal ofUrology 1986; 136(4): 861863.

16-Shankar KR, Losty PD, Hopper M, Wong L, Rickwood AMK: Outcome of hypospadias fistula repair. $B J U$ International 2002; 89(1): 103-105.

17-Routh JC, Wolpert JJ, Reinberg Y: Tunneled tunica vaginalis flap for recurrent urethrocutaneous fistulas. Adv Urol2008; 176: $1578-1580$.

18-Sunay M, Dadali M, Karabulut A, Emir L, Erol D: Our 23-year experience in urethrocutaneous fistulas developing after 
hypospadias surgery. Urology 2007; 69: 366-368.

19-Uday S: Chatterjee, Manas K Mandai, Supriyo Basu, Ranjit Das, Tapas Majhi: Comparative study of Dartos fascia and tunica vaginalis pedicle wrap for the tubularized incised plate in primary hypospadias repair. BJU International 2004; 1102-1104.
20-Muruganandham K, Ansari MS, Dubey D, Mandhani A, Srivastava A, et al: Urethrocutaneous fistula after hypospadias repair: Outcome of three types of closure techniques. Pediatric Surg Int 2010;26(3): 305-308. 